PROCEEDINGS OF THE

AMERICAN MATHEMATICAL SOCIETY

Volume 137, Number 12, December 2009, Pages 4043-4048

S 0002-9939(09)10026-6

Article electronically published on August 7, 2009

\title{
ALMOST COMMUTING UNITARIES WITH SPECTRAL GAP ARE NEAR COMMUTING UNITARIES
}

\author{
TOBIAS J. OSBORNE
}

(Communicated by Marius Junge)

\begin{abstract}
Let $\mathcal{M}_{n}$ be the collection of $n \times n$ complex matrices equipped with operator norm. Suppose $U, V \in \mathcal{M}_{n}$ are two unitary matrices, each possessing a gap larger than $\Delta$ in their spectrum, which satisfy $\|U V-V U\| \leq \epsilon$. Then it is shown that there are two unitary operators $X$ and $Y$ satisfying $X Y-Y X=0$ and $\|U-X\|+\|V-Y\| \leq E\left(\Delta^{2} / \epsilon\right)\left(\frac{\epsilon}{\Delta^{2}}\right)^{\frac{1}{6}}$, where $E(x)$ is a function growing slower than $x^{\frac{1}{k}}$ for any positive integer $k$.
\end{abstract}

\section{INTRODUCTION}

An old problem from the 1950s, popularised by Halmos, asks: if a pair $\{A, B\}$ of matrices almost commute, then are they necessarily close to a pair $\left\{A^{\prime}, B^{\prime}\right\}$ of commuting matrices [14, 3, 1, 7, 13]? Voiculescu realised that this is not necessarily the case and presented a family of pairs of unitary matrices which asymptotically commute, but which were far from any commuting pair of matrices [15].

Since this time there has been considerable work on this problem culminating in 1995 with the proof of Lin that for any pair $\{A, B\}$ of hermitian matrices which satisfy $\|A B-B A\| \leq \epsilon$, with $\epsilon>0$ and $\|A\|,\|B\| \leq 1$, there exists a hermitian pair $\left\{A^{\prime}, B^{\prime}\right\}$ of matrices and $\delta(\epsilon)>0$ such that $\left\|A^{\prime}-A\right\|+\left\|B^{\prime}-B\right\| \leq \delta(\epsilon)$ [ [see [6] for a simplified exposition). The proof was nonconstructive, and the dependence of $\delta$ on $\epsilon$ was not quantified, apart from the nontrivial fact that $\delta$ did not depend on the size of the matrices. Recently, Hastings [8] presented a new constructive proof of the result of Lin and was able to calculate quantitative bounds.

In light of the recent results of 8 it is worthwhile to reconsider the problem of when a pair of almost commuting unitary matrices are near a pair of commuting unitaries. There have been several studies of this problem, partially motivated by Voiculescu's original counterexample, and it was quickly realised that there was, in general, a $K$-theoretic obstruction [4, 5. It was shown that when this obstruction vanishes, a pair of almost commuting unitaries is indeed close to a commuting pair 10, 11. Again, the proof was nonconstructive, and quantitative bounds were not provided. In this paper we partially ameliorate this situation by proving the following.

Received by the editors September 15, 2008, and, in revised form, April 18, 2009.

2000 Mathematics Subject Classification. Primary 15A15, 15A27, 47A55; Secondary 47B47.

(C)2009 American Mathematical Society

Reverts to public domain 28 years from publication 
Theorem 1.1. Let $U$ and $V$ be two unitary operators such that

$$
\|[U, V]\| \leq \epsilon,
$$

where $[U, V]=U V-V U$, and also such that there exist $0<\Delta_{1}<\pi$ and $0<\Delta_{2}<\pi$ with ${ }^{1}$

$$
\operatorname{spec}(U) \subset S^{1} \backslash\left[-\Delta_{1}, \Delta_{1}\right] \quad \text { and } \operatorname{spec}(V) \subset S^{1} \backslash\left[-\Delta_{2}, \Delta_{2}\right] .
$$

Then there exist two commuting unitary operators $X$ and $Y$ such that

$$
\|U-X\| \leq \delta\left(\epsilon /\left(\Delta_{1} \Delta_{2}\right)\right) \quad \text { and } \quad\|V-Y\| \leq \delta\left(\epsilon /\left(\Delta_{1} \Delta_{2}\right)\right),
$$

where $\delta(x)=E(1 / x) x^{\frac{1}{6}}$ and $E(x)$ grows slower than $x^{1 / k}$ for any positive integer $k$.

The proof of Theorem 1.1 is based on three observations. The first is that the principle branch of the matrix logarithm of a unitary matrix $U$ with spectral gap can be represented in terms of a rapidly convergent Laurent series in $U$. The second observation is that the matrix logarithms $\{A, B\}$ of a pair $\{U, V\}$ of approximately commuting unitaries must themselves be approximately commuting, so they are close to a pair of commuting matrices $\left\{A^{\prime}, B^{\prime}\right\}$. The final observation is that these commuting matrices give rise to a commuting pair $\{X, Y\}$ of unitaries close to $\{U, V\}$.

It is worth noting that the result proved in this paper does depend on the presence of a spectral gap in the spectrum of $U$ and $V$. It is rather plausible that this is not the strongest result possible: if the $K$-theoretic obstruction of 4, 5] vanishes, it should be the case that a quantitative bound on $\delta$, in the spirit of Hastings, can be given generalising the original result of [10, 11]. Such a result shouldn't depend on the presence of a gap in the spectrum of $U$ and $V$. However, at the current time, it is unclear how to use the arguments of Hastings in 8 , to prove such a quantitative version.

\section{LOGARITHMS OF UNITARY MATRICES WITH SPECTRAL GAP}

Theorem 2.1. Let $U \in \mathcal{M}_{n}$ be a unitary matrix such that there exists a gap $\Delta$ with $0<\Delta<\pi$ such that

$$
\operatorname{spec}(U) \subset S^{1} \backslash[-\Delta, \Delta] .
$$

Then the principle branch of the matrix logarithm of $U$ may be represented as a Laurent series

$$
\log (U)=-i \sum_{k=-\infty}^{\infty} c_{k} U^{k}
$$

where $\left|c_{k}\right| \leq \min \left\{\pi, C /\left(\Delta k^{4}\right)\right\}$ and $C$ is constant.

Remark 2.2. This result is adapted from a physical argument developed in 12 .

Proof. We begin by calculating the eigenvalues and eigenvectors for $U$ :

$$
U v_{j}=e^{i \phi_{j}} v_{j}
$$

where $v_{j}$ are the eigenvectors of $U$ and we choose $\phi_{j} \in[0,2 \pi$ ). (If the gap in $U$ 's spectrum was not centred on 0 , we could, by multiplying by an overall phase $e^{i \zeta} \mathbb{I}$,

\footnotetext{
${ }^{1}$ We denote the unit circle in the complex plane by $S^{1}$. Where obvious, we identify subintervals of $[-\pi, \pi)$ with their image in $S^{1}$ under the map $e^{i \phi}$.
} 
$\zeta \in \mathbb{R}$, arrange for the zero of angle to lie at the origin. Such a gap always exists for finite dimensional unitary matrices, but not necessarily for infinite operators.)

We want to find a hermitian matrix $H$ so that $U=e^{i H}$ and $H v_{j}=\phi_{j} v_{j}$. To do this we suppose that

$$
H=\sum_{k=-\infty}^{\infty} d_{k} U^{k}
$$

and we solve for the coefficients $d_{k}$ : by applying both sides of the above equation to the common eigenvector $v_{j}$, we find that

$$
\phi_{j}=\sum_{k=-\infty}^{\infty} d_{k} e^{i k \phi_{j}}
$$

Hence, if we can find $d_{k}$ such that

$$
\theta=\sum_{k=-\infty}^{\infty} d_{k} e^{i k \theta}
$$

for all $\theta \in[0,2 \pi)$, then we are done. (Recall that we've arranged it so there are no eigenvalues of $U$ at the point $\theta=0$.) To solve for $d_{k}$ we integrate both sides of (2.6) with respect to $\theta$ over the interval $[0,2 \pi)$ against $\frac{1}{2 \pi} e^{-i l \theta}$, for $l \in \mathbb{Z}$ :

$$
\frac{1}{2 \pi} \int_{0}^{2 \pi} \theta e^{-i l \theta} d \theta=\frac{1}{2 \pi} \sum_{k=-\infty}^{\infty} d_{k} \int_{0}^{2 \pi} e^{i(k-l) \theta} d \theta .
$$

Thus we learn that the $d_{k}$ are nothing but the Fourier coefficients of the periodic sawtooth function $f(\theta+2 \pi l)=\theta, \theta \in[0,2 \pi), l \in \mathbb{Z}$ :

$$
d_{k}= \begin{cases}\pi, & k=0 \\ \frac{i}{k}, & k \neq 0\end{cases}
$$

Unfortunately the sawtooth wave has a jump discontinuity, and hence the Fourier series is only conditionally convergent. The way to proceed is to assume that we have some further information, namely, that $U$ has a gap $\Delta$ in its spectrum.

The idea now is to exploit the existence of the gap to provide a more useful series representation for $H$. We do this by calculating the Fourier coefficients $c_{k}$ of the sawtooth wave $f(\theta)$ convolved with a sufficiently smooth smearing function $\chi_{\gamma}(\theta)$; the Fourier series inherits a better convergence from the smoothness properties of the smearing function. That is, we define $c_{k}$ to be the Fourier coefficients of

$$
g(\theta)=\left(f \star \chi_{\gamma}\right)(\theta)=\int_{-\infty}^{\infty} f(\theta-y) \chi_{\gamma}(y) d y .
$$

We choose $\chi_{\gamma}$ to be the function

$$
\chi_{\gamma}(x)= \begin{cases}\left(1-\left(\frac{x}{\gamma}\right)^{2}\right)^{3}, & |x| \leq \gamma \\ 0, & |x|>\gamma\end{cases}
$$

The Fourier transform $\widehat{\chi}_{\gamma}(t) \equiv \frac{1}{\sqrt{2 \pi}} \int_{-\infty}^{\infty} \chi_{\gamma}(x) e^{i x t} d x$ satisfies

$$
\left|\widehat{\chi}_{\gamma}(t)\right| \leq \frac{C}{\gamma t^{3}}
$$


where $C$ is a constant. Note that, as a consequence of the compact support of $\chi_{\gamma}(y), g(\theta)=f(\theta), \forall \theta \in(\gamma, 2 \pi-\gamma)$. An application of the convolution theorem then tells us that the Fourier coefficients $d_{k}$ are given by

$$
c_{k}=\widehat{\chi}_{\gamma}(k) d_{k} .
$$

Choosing $\gamma<\Delta$ allows us to conclude that

$$
H=\sum_{k=-\infty}^{\infty} c_{k} U^{k}
$$

because both $f(\theta)$ and $g(\theta)$ agree on the spectrum of $U$. According to (2.11) we now have that $\left|c_{k}\right| \leq \min \left\{\pi, C /\left(\Delta k^{4}\right)\right\}$.

\section{Constructing commuting Logarithms}

In this section we exploit the following theorem of Hastings.

Theorem 3.1 ([8]). Let $A$ and $B$ be Hermitian, $n \times n$ matrices, with $\|A\|,\|B\| \leq 1$. Suppose $\|[A, B]\| \leq \epsilon$. Then there exist Hermitian $n \times n$ matrices $A^{\prime}$ and $B^{\prime}$ such that

(1) $\left[A^{\prime}, B^{\prime}\right]=0$ and

(2) $\left\|A^{\prime}-A\right\| \leq \delta(\epsilon)$ and $\left\|B^{\prime}-B\right\| \leq \delta(\epsilon)$, with

$$
\delta(\epsilon)=E(1 / \epsilon) \epsilon^{\frac{1}{6}},
$$

where the function $E(x)$ grows slower than $x^{\frac{1}{k}}$ for any positive integer $k$. The function $E(x)$ does not depend on $n$.

Proof of Theorem 1.1. The first step of the proof is to exploit the presence of the spectral gap and use Theorem 2.1 to represent the logarithms $A$ and $B$ of $U=e^{i A}$ and $V=e^{i B}$ via Laurent series with fast decay:

$$
A=\sum_{j=-\infty}^{\infty} c_{j} U^{j}
$$

and

$$
B=\sum_{k=-\infty}^{\infty} d_{k} V^{k},
$$

with

$$
\left|c_{j}\right| \leq \min \left\{\pi, C /\left(\Delta_{1} j^{4}\right)\right\}, \quad\left|d_{k}\right| \leq \min \left\{\pi, C /\left(\Delta_{2} k^{4}\right)\right\},
$$

where $C$ is a constant.

Now that we have an expression for $A$ and $B$ we can work out the norm of their commutator using the Leibniz property via

$$
\begin{aligned}
{[A, B] } & =\sum_{j=-\infty}^{\infty} \sum_{k=-\infty}^{\infty} c_{j} d_{k}\left[U^{j}, V^{k}\right] \\
& =\sum_{j=-\infty}^{\infty} \sum_{k=-\infty}^{\infty} \sum_{m=1}^{j} \sum_{n=1}^{k} c_{j} d_{k} V^{m-1} U^{n-1}[U, V] U^{j-n} V^{k-m}
\end{aligned}
$$


and then taking the norm of both sides. Applying the triangle inequality and the unitary invariance of the operator norm gives us

$$
\begin{aligned}
\|[A, B]\| & \leq \sum_{j=-\infty}^{\infty} \sum_{k=-\infty}^{\infty} j k\left|c_{j}\right|\left|d_{k}\right|\|[U, V]\| \leq \epsilon\left(\sum_{j=-\infty}^{\infty} j\left|c_{j}\right|\right)\left(\sum_{k=-\infty}^{\infty} k\left|c_{k}\right|\right) \\
& \leq \epsilon \frac{\alpha}{\Delta_{1} \Delta_{2}}
\end{aligned}
$$

where $\alpha$ is a constant coming from $C^{2}$ and the summation of the separate infinite series.

The next step is to apply Theorem 3.1 to construct two commuting operators $A^{\prime}$ and $B^{\prime}$ such that

$$
\left\|A^{\prime}-A\right\| \leq \delta\left(\epsilon \alpha / \Delta_{1} \Delta_{2}\right) \quad \text { and } \quad\left\|B^{\prime}-B\right\| \leq \delta\left(\epsilon \alpha / \Delta_{1} \Delta_{2}\right)
$$

and then, via exponentiation, we define $U^{\prime}=e^{i A^{\prime}}$ and $V^{\prime}=e^{i B^{\prime}}$. The distance between $U^{\prime}$ and $U$ can be bounded as follows (following [2], p. 252):

$$
\left\|U^{\prime}-U\right\| \leq \int_{0}^{1}\left\|A^{\prime}-A\right\| d s \leq \epsilon,
$$

and similarly for $V^{\prime}$. Redefining $E(x)$ gives the result.

\section{REFERENCES}

1. I. David Berg and Kenneth R. Davidson, Almost commuting matrices and the Brown-DouglasFillmore theorem, Bull. Amer. Math. Soc. (N.S.) 16 (1987), no. 1, 97-100. MR866023 (88a:47022)

2. Ola Bratteli and Derek W. Robinson, Operator algebras and quantum statistical mechanics. 2, 2nd ed., Texts and Monographs in Physics, Springer-Verlag, Berlin, 1997. MR.98e:82004

3. Kenneth R. Davidson, Almost commuting Hermitian matrices, Math. Scand. 56 (1985), no. 2, 222-240. MR813638 (87e:47012)

4. Ruy Exel and Terry Loring, Almost commuting unitary matrices, Proc. Amer. Math. Soc. 106 (1989), no. 4, 913-915. MR.975641 (89m:15003)

5. Ruy Exel and Terry A. Loring, Invariants of almost commuting unitaries, J. Funct. Anal. 95 (1991), no. 2, 364-376. MR1092131 (92a:46083)

6. Peter Friis and Mikael Rørdam, Almost commuting self-adjoint matrices-a short proof of Huaxin Lin's theorem, J. Reine Angew. Math. 479 (1996), 121-131. MR.1414391 (97i:46097)

7. P. R. Halmos, Some unsolved problems of unknown depth about operators on Hilbert space, Proc. Roy. Soc. Edinburgh Sect. A 76 (1976/77), no. 1, 67-76. MR0451002 (56:9292)

8. M. B. Hastings, Making Almost Commuting Matrices Commute, arXiv:0808.2474, 2008.

9. Hua Xin Lin, Almost commuting unitary elements in purely infinite simple $C^{*}$-algebras, Math. Ann. 303 (1995), no. 4, 599-616. MR1359951 (96k:46101)

10. Huaxin Lin, Almost commuting selfadjoint matrices and applications, Operator Algebras and Their Applications (Waterloo, ON, 1994/1995), Fields Inst. Commun., vol. 13, Amer. Math. Soc., Providence, RI, 1997, pp. 193-233. MR.1424963 (98c:46121)

11. _ Almost commuting unitaries and classification of purely infinite simple $C^{*}$-algebras, J. Funct. Anal. 155 (1998), no. 1, 1-24. MR1622808 (99e:46070)

12. Tobias J. Osborne, Approximate locality for quantum systems on graphs, Phys. Rev. Lett. 101 (2008), no. 14. MR 2460715

13. Peter Rosenthal, Research Problems: Are Almost Commuting Matrices Near Commuting Matrices?, Amer. Math. Monthly 76 (1969), no. 8, 925-926. MR.1535586 
14. Stanisław J. Szarek, On almost commuting Hermitian operators, Proceedings of the Seventh Great Plains Operator Theory Seminar (Lawrence, KS, 1987), Rocky Mountain J. Math. 20 (1990), 581-589. MR.1065854 (91f:47032)

15. Dan Voiculescu, Asymptotically commuting finite rank unitary operators without commuting approximants, Acta Sci. Math. (Szeged) 45 (1983), no. 1-4, 429-431. MR708811 (85d:47035)

Department of Mathematics, Royal Holloway, University of London, Egham, TW20 0EX, United Kingdom

E-mail address: tobias.osborne@rhul.ac.uk 